\title{
Noise Removal in ECG Signal using Windowing Technique and its Optimization
}

\author{
Priyanka $^{1 *}$ and Gurjit Kaur ${ }^{2}$ \\ School of Information and Communication Technology, Gautam Buddha University, India
}

Submission: July 24, 2017; Published: August 29, 2017

*Corresponding author: Priyanka, School of Information and Communication Technology, Gautam Buddha University, Greater Noida, Uttar Pradesh, India, Email: priyanka.vishwakarma13@gmail.com

\begin{abstract}
ECG signal is widely used for detection and diagnosis of various heart related diseases. Feature extraction through ECG is a new application that is rapidly growing these days. While acquiring ECG signal, it gets contaminated to a number of sources with various types of artifacts such as baseline wander interference, motion artifact, instrumentation noise, electrode contact noise, EMG noise etc. In this research work, different windowing technique to remove noise in corrupted ECG signal has been analysed through system model. The windows used were Kaiser, Rectangle, Hamming, Hanning and Welch window. The output was analysed and compared using MSE, SNR, Positive Peak and THD performance parameters for power line interference, muscle noise and EMG noise. This research work gives an optimal ECG noise removal windowing system that concludes which particular window should be applied for a particular type of noise. Thus, this research concludes that Kaiser window has best denoising performance followed by Rectangle window and in some cases, Hamming window also. Moreover, all the windows have been optimized for best sampling rate.
\end{abstract}

Abbreviations: ECG: Electro cardio graphy; MSE: Mean Square Error; SNR: Signal to Noise Ratio; THD: Total Harmonic Distortion

\section{Introduction}

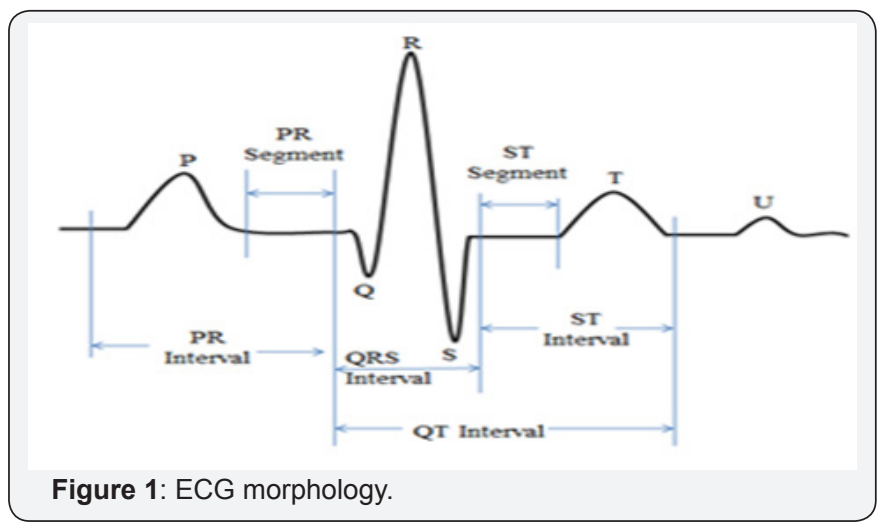

Biomedical signals are always polluted with some kind of noise that corrupts the signal and leads to improper detection accounting for mistakes in recognizing details for diagnostic purposes. Thus, signal has to be pre-processed to eliminate unwanted disturbances encountered in clinical environment which is the concept of noise removal. Noise removal helps to ensure the fidelity of the signal for proper diagnosis. The sources of noise can be plenty like respiratory movements, power line frequency interference $(50 / 60 \mathrm{~Hz})$, random body movements, instrumentation noise, noise due to improper electrode placement and so on. ECG is a widely used tool to understand the heart condition $[1,2]$. The ECG signal is an electrical illustration of the activity of human heart. Computerized ECG analysis is commonly used as a reliable technique for the diagnosis of heart diseases. It is acquired by placing electrodes on the skin of the patient. The ECG morphology consist of $\mathrm{P}$ wave, $\mathrm{Q}$ wave, $\mathrm{R}$ wave, $\mathrm{S}$ wave, $\mathrm{U}$ wave, $\mathrm{QRS}$ complex and various other segments as shown in the Figure 1.

The artifacts contaminating ECG signal are namely electrode contact noise, powerline interference, motion artifacts, baseline wandering, EMG noise, instrumentation noise generated by electronics devices. Noise removal in ECG signal is complicated due to the time varying nature of ECG signals. Many researchers had adopted different method for removing the noise from ECG signal. Jacek M Leski et al. [3] presented a new class of nonlinear filter bank for reducing the noise in the ECG signal using different types of filters like Moving average filter, linearphase FIR filter using rectangular and Kaiser windows, Adaptive filter and Nonlinear filter. Seema Rani et al. [4] presented a comparison between Digital FIR and IIR filter complexity for removal of baseline noise in ECG signal in which though IIR filters required less computational power with the introduction of phase distortion and FIR filters introduced ringing effect. Rinky Lakhwani et al. [5] proposed a method for the removal of baseline wandering in ECG signal and designed a digital FIR filters using different windowing techniques which are rectangular, Gaussian, hamming and Kaiser. Yuta Kondo et al. [6] developed a system that detects changes in R-R interval 
fluctuation which is related to stress and drowsiness of the driver, and alert the driver. The developed system measures ECG with a contact electrode on the steering wheel and a capacitively coupled electrode on the driver's seat. Nidhi Rastogi et al. [7] developed a new technique for removal of baseline wander based on Daubechies wavelet decomposition and also integrated adaptive thresholding techniques and Savitzky-Golay filtering and compared their novel method to the conventional one.

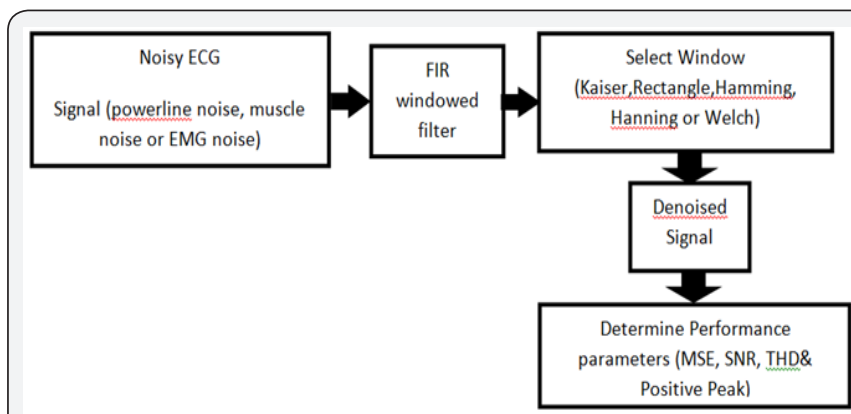

Figure 2: Block diagram of the system model design.

Till date, none of the researchers have compared/optimized various noisy signals using different windowing techniques. In this research paper, we have analysed the performance of ECG noise removal system by considering power line noise, muscle noise and EMG noise using Kaiser, Rectangle, Hamming, Welch and Hanning windowing techniques. The output is analysed and compared for MSE, SNR, Positive Peak and THD performance parameters. The research work gives an optimal ECG noise removal system which concludes that which particular windowing technique should be applied for a particular noise. Now, Paper is organized as follows. Section II describes the various research works done in past by different researchers. Section III discusses about the experimental results obtained, waveforms generated and graph analysis of different performance parameters. Section IV presents the conclusion of our research paper (Figure 2).

\section{System design and results}

Table 1: Design Parameters of ECG signal and filter.

\begin{tabular}{|c|c|}
\hline Parameters & ECG Values \\
\hline Filter Type & Lowpass \\
\hline Lower cut-off frequency & $0.12 \mathrm{~Hz}$ \\
\hline Higher cut-off frequency & $0.45 \mathrm{~Hz}$ \\
\hline Sampling frequency & $4 \mathrm{~Hz}$ \\
\hline Taps & 25 \\
\hline Output upper limit & $1.2 \mathrm{mV}$ \\
\hline Output lower limit & $-0.4 \mathrm{mV}$ \\
\hline Heart rate & $60 \mathrm{bpm}$ \\
\hline Sampling Rate & $512 \mathrm{~Hz}$ \\
\hline
\end{tabular}

A denoising system has been designed by us in order to achieve the noise removal of ECG signal. Signal corrupted by various types noises were taken and denoised using different windowing techniques in an FIR filter such as Kaiser window, Rectangle window, Hamming window, Hanning window and Welch window. The performance of each window is seen and optimization of operating frequency is carried out for best denoised output. The sampling frequency for each window obtained after optimization is used to eliminate various noises that corrupt the ECG signal. In this research paper we have taken three different types of noisy ECG signal and denoised them using the system model defined above and also design parameters of ECG and filters are defined by us in Table 1 in order to achieve best results. Parameters like MSE, THD, SNR and Positive peak were evaluated which help us to determine the performance of the system for a particular window.

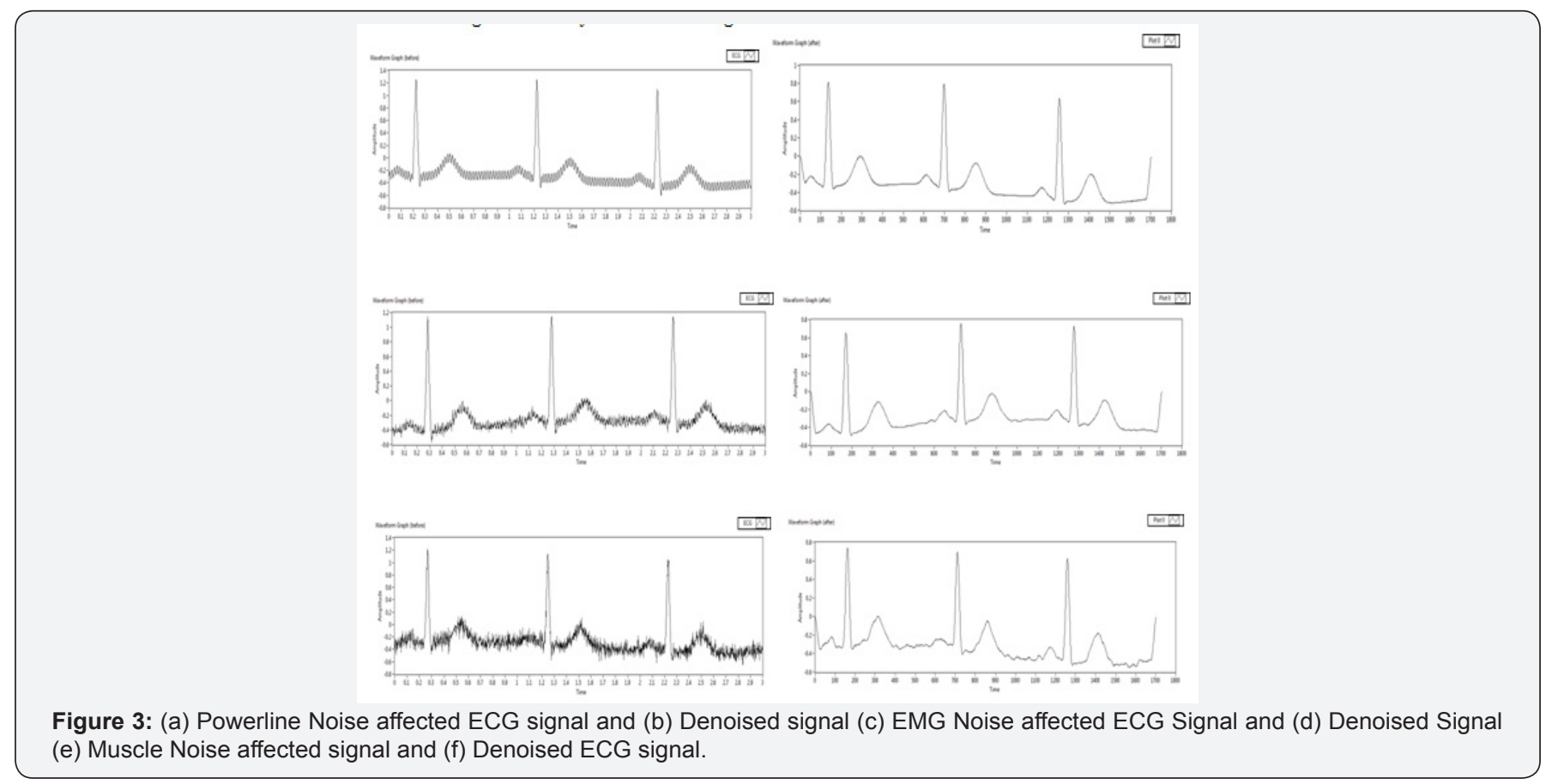


An ECG signal affected by power line noise as shown in Figure 3a was input to the system model designed. The output of the system when filtering technique with various windows was used gave the denoised signal with reduced amount of noise and satisfactory level of signal peak as shown in the Figure 3b. An ECG signal affected by muscle noise as shown in Figure 3c was input to the system model designed. The output of the system when filtering technique with various windows was used gave the denoised signal with reduced amount of noise and satisfactory level of signal peak as shown in the Figure 3d. An ECG signal affected by EMG noise as shown in Figure 3e was input to the system model designed. The output of the system when filtering technique with various windows was used gave the denoised signal with reduced amount of noise and satisfactory level of signal peak as shown in the Figure 3f.

\section{Analysis of different performance parameters for power line noise}
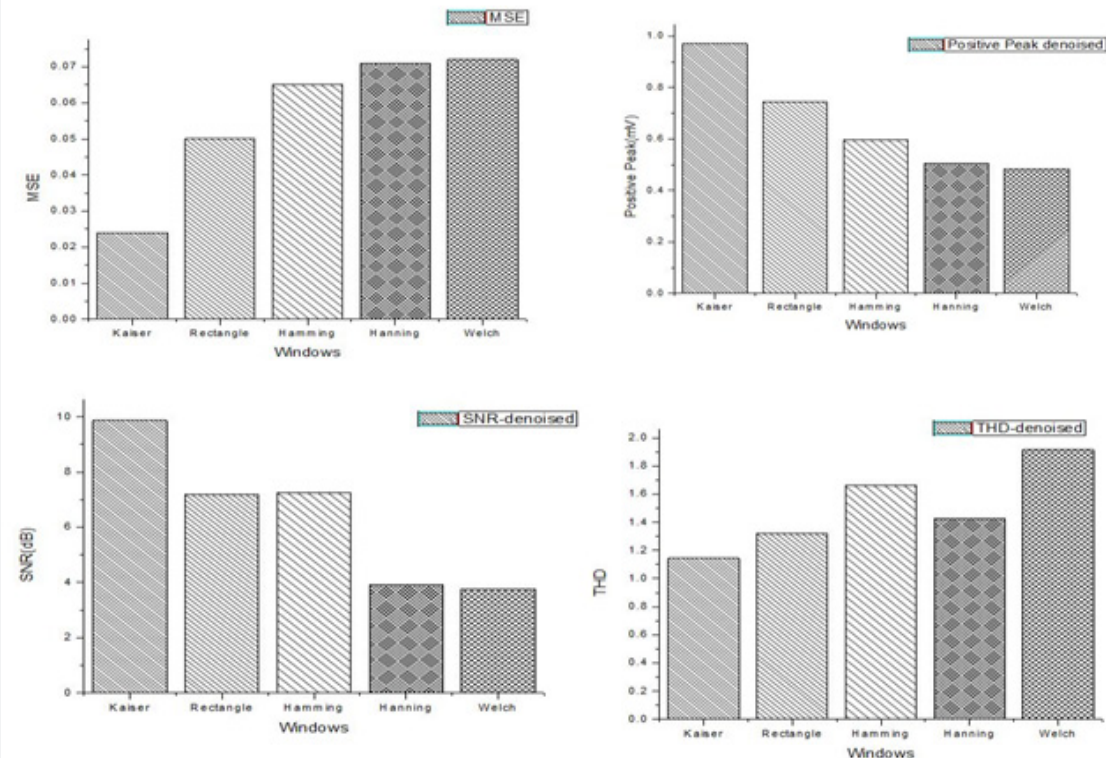

Figure 4: (a) Analysis of MSE for Powerline noise (b) Analysis of Positive-Peak for Powerline noise (c) Analysis of SNR for Powerline noise \& (d) Analysis of THD for Powerline noise.

Figure 4a a low value of MSE is required for good performance which is shown by Kaiser window with value 0.024. Rectangle window has higher MSE that Kaiser window and Hamming window in turn has highest of the two. The values of MSE obtained through Hamming, Hanning and Welch window is much higher and unsatisfactory. Thus, it can be concluded that Kaiser and Rectangle window are suitable for denoising considering MSE only. Figure 4b positive peak is intended to be high and such is obtained from Kaiser window with value $0.9699 \mathrm{mV}$. But all other windows show peak values less than $0.7 \mathrm{mV}$ which is not suitable for good denoising performance evaluation. Thus, only Kaiser window proves to be paramount in retention of positive peak. Figure 4c in this graph, Kaiser window shows highest SNR value $9.864 \mathrm{~dB}$ after denoising which is advantageous. Though, Rectangle and Hamming windows show lesser SNR values $7.189 \mathrm{~dB} \& 7.264 \mathrm{~dB}$ they individually have acceptable levels of SNR. Thus, it can be concluded that all the three windows are suitable when SNR is considered. Hanning and Welch windows on the other hand give extremely poor results which are not even half of the original signal values $3.922 \mathrm{~dB} \& 3.767 \mathrm{~dB}$. Figure $4 \mathrm{~d}$ as shown in the above graph, THD values $1.1436 \& 1.3213$ of Kaiser window and Rectangle window are close to each other and also lesser than those of the rest. Although, Kaiser window is preferred over Rectangle window in terms of THD. The THD values of Hamming, Hanning and Welch window are not good wherein Welch window has the highest THD value 1.9144 of all which is not suitable.

\section{Analysis of different performance parameters for muscle noise}

From the Figure 5 a Graph shows that Kaiser window has the least MSE of 0.047. Rectangle and Hamming window have close resemblance in performance with MSE higher than that of Kaiser window. But, Hanning and Welch window have high MSE values $0.069 \& 0.071$ which are not suitable for good performance in denoising. From the Figure $5 \mathrm{~b}$ graph shows that Kaiser and Rectangle window has good values of positive peaks and other three windows i.e. Hamming, Hanning and Welch window with values $0.6152 \mathrm{mV}, 0.5836 \mathrm{mV}$ and $0.5752 \mathrm{mV}$ respectively have lower levels of peak values which is proves to be degrading to the system. Thus, for retention of positive peak, only Kaiser and Rectangle windows are to be used. From the above Figure $5 \mathrm{c}$ it is evident that Kaiser, Rectangle and Hamming windows have highest values of denoised SNR i.e. of about 7.5dB. However, if analysed individually, Hanning and Welch windows too have good values of SNR independently. Surprisingly, Welch window performs better in giving acceptable SNR values of denoised signals. Thus it can be concluded that all the windows perform 
nicely in providing amplification of SNR in denoised signals. From the Figure $5 \mathrm{~d}$ graph depicts that Kaiser and Rectangle window have the least THD value among all which is a desirable feature. Hamming window gives slightly greater THD than Kaiser and Rectangle window whereas Hanning and Welch windows give unacceptably high THD values. Thus, we can conclude that though Hamming window shows comparable results with Kaiser and Rectangle window, it will not be practically preferred over the two because of the relatively slightly degrading performance.
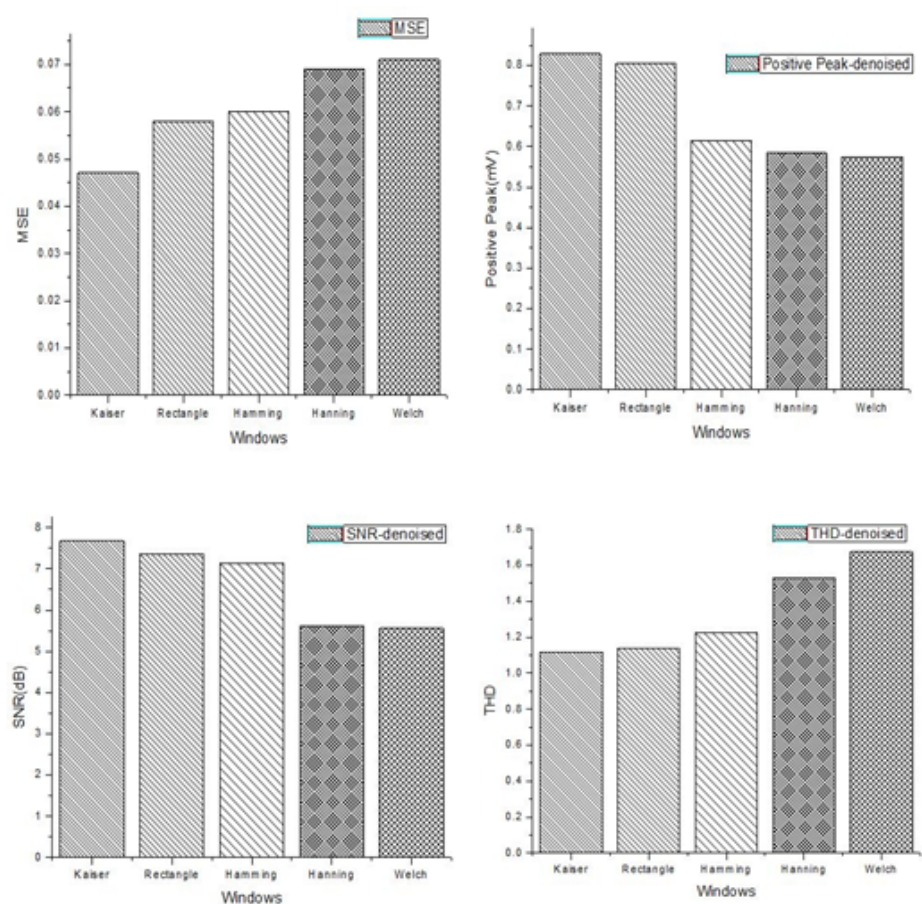

Figure 5: (a) Analysis of MSE for Muscle noise (b) Analysis of Positive Peak for Muscle noise (c) Analysis of SNR for Muscle noise \& (d) Analysis of MSE for Muscle noise.

\section{Analysis of different performance parameters for EMG noise}
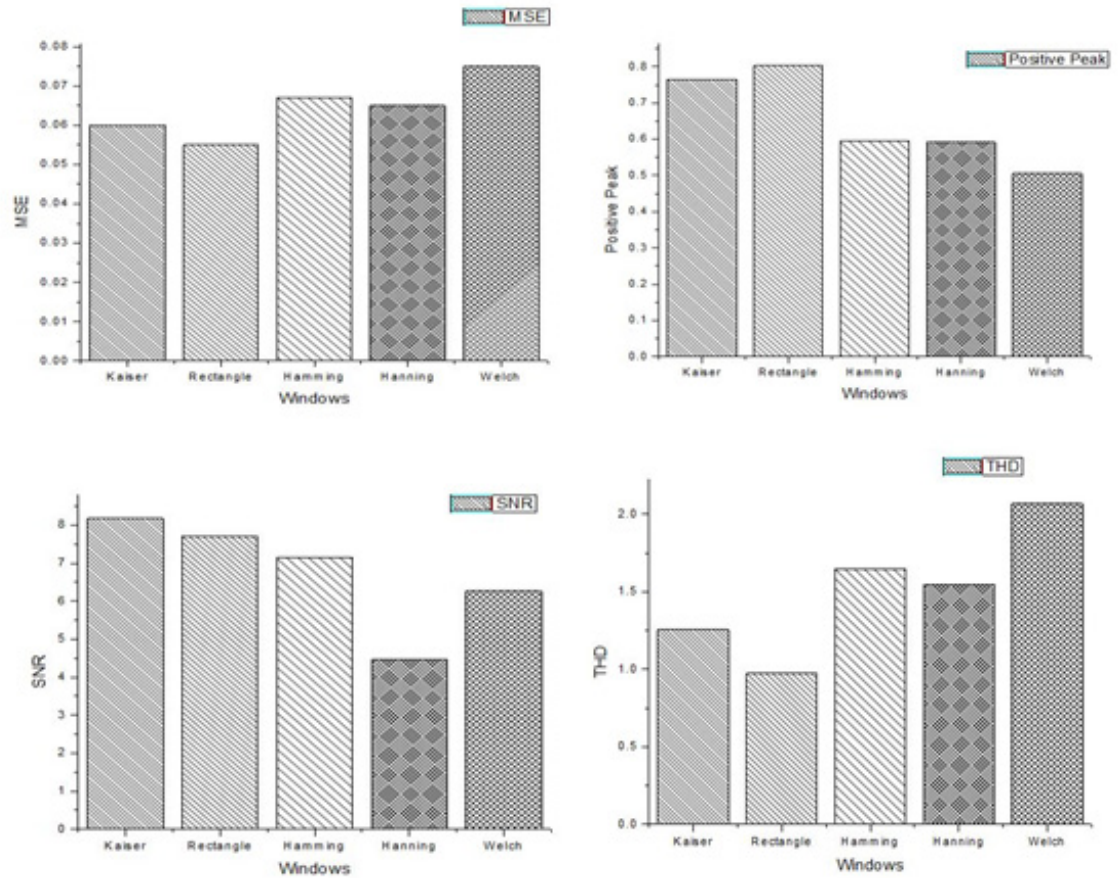

Figure 6: (a) Analysis of MSE for EMG noise (b) Analysis of Positive Peak for EMG noise (c) Analysis of SNR for EMG noise \& (d) Analysis of THD for EMG noise. 


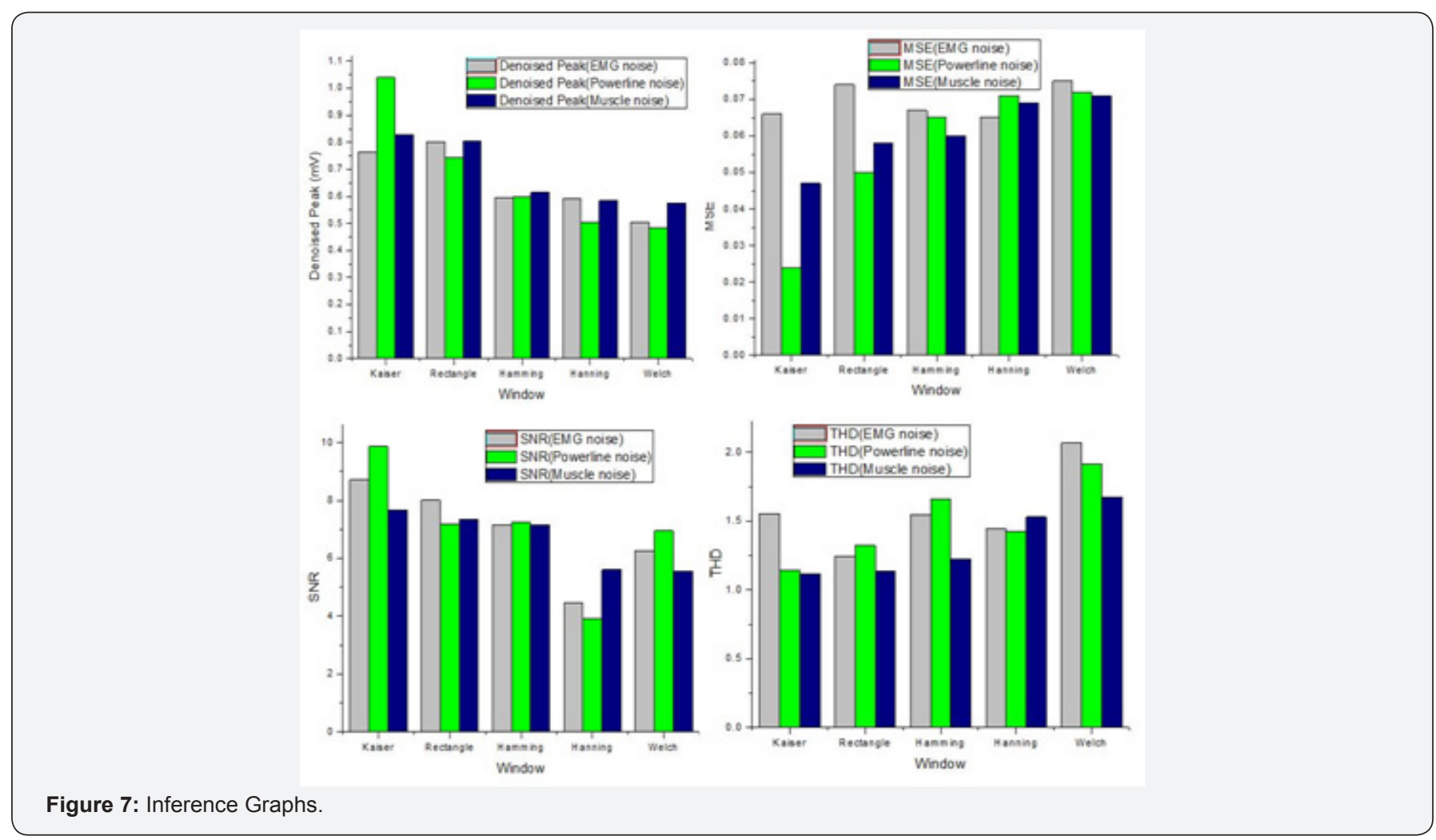

From the Figure 6a graph depicted that Kaiser and Rectangle window have low MSE values wherein Rectangle has the least one. Though Hamming and Hanning window have comparable values to the former two windows, their individual values are not appropriate according to our system model. Welch window on the other hand has the highest MSE value of all which is detrimental. From the Figure 6b, both Kaiser and Rectangle window show good levels of positive peaks with the values $0.7645 \mathrm{mV}$ and $0.8023 \mathrm{mV}$ respectively. Amongst the two, a Rectangle window showed better result which concludes that it is superior to Kaiser window. All the other windows show unacceptable positive peaks with values less than $0.6 \mathrm{mV}$. This implies that more information is being lost with the use of these windows. From the Figure $6 \mathrm{c}$ Kaiser window proves to be best with the value of $8.173 \mathrm{~dB}$.

Moreover, Rectangle window also follows Kaiser window closely with SNR value of $8.005 \mathrm{~dB}$. Hamming and Welch window also show average SNR values of $7.138 \mathrm{~dB}$ and $6.259 \mathrm{~dB}$ which is considerable in some applications. Hanning window shows unsatisfactory results. From the above Figure $6 \mathrm{~d}$, we can analyse that Rectangle window has the least THD value of 1.2451 and Kaiser window also has somewhat acceptable THD value but it is more than that of the Rectangle window. Moreover, all the other three windows show unacceptable values of THD which are highly distorted than our original input signal. This means that distortion is increasing which is not a preferable factor (Figure 7).

\section{Conclusion}

In this paper, different windowing techniques are applied on the noisy ECG signal in order to remove noise from it. Different windowing techniques used were Kaiser, Rectangle, Hamming, Hanning and welch window and output was evaluated using performance parameters such as MSE, SNR, Positive Peak and THD. During the analysis of power line noise, it was seen that Kaiser window showed best performance among all the chosen performance parameters. This was closely followed by Rectangle window that also showed good results in all performance parameters except for SNR. Therefore, majorly it can be said that both Kaiser and Rectangle window performed satisfactorily for removing power line noise from the ECG signal. Also, when considering THD specifically Hanning window showed good results. When muscle noise was analysed, it was seen that Kaiser and Rectangle window showed best results for all the chosen performance parameters. Hamming window showed very close resemblance to these two windows and to rectangle window in particular. Therefore, it can be concluded that these three windows are efficient in removing muscle noise from the ECG signal. So, depending on the application any of these three windows can be considered. When analysing EMG noised affected ECG signal, majorly Rectangle window showed good results along with Kaiser window that showed satisfactory results. This implies that for removing EMG noise from ECG signal, Rectangle window is preferred over Kaiser window unlike the trend in other two noises. Thus, it can be concluded that Kaiser window has best denoising performance followed by Rectangle window 
and in some cases Hamming window also. In this research work, all the windows have been optimized for best sampling rate.

\section{Future Scope}

Future scope of this research is: All these windows can be further optimized for different system parameters. The use of Wavelet denoising technique can also be considered. It can be realized for real-time systems.

\section{References}

1. Kaiser JF (1974) Non recursive digital filter design using the I0- sinh window function. Proc 1974 IEEE® symp circuits and systems.

2. Landernas K (2004) Implementation of digital filters using digitalseries arithmetics. Department of electronics, Licentiate thesis No. 23.

3. Leski JM, Henzel N (2005) ECG Baseline wander and powerline interference reduction using nonlinear filter bank. Signal Processing 85(4): 781-793.

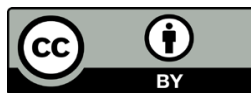

This work is licensed under Creative Commons Attribution 4.0 Licens

DOI: 10.19080/AIBM.2017.06.555676
4. Rani S, Kaur A, Ubhi JS (2011) Comparative study of FIR and IIR filters for the removal of Baseline noises from ECG signal. International Journal of Computer Science and Information Technologies 2(3): 1105-1108.

5. Lakhwani R, Ayub S, Saini JP (2013) DOI 10.1109, 2013 Design and Comparison of Digital Filters for Removal of Baseline Wandering from ECG Signal ${ }^{{ }^{\text {th }}}$ International Conference on Computational Intelligence and Communication Networks, IEEE.

6. Kondo Y, Sasaki K, Nakano Y, Sano S (2010) Reduction of baseline fluctuation in electrocardiogram measurement for a car driver. IEEE DOI: 978-1-4244- 6890-4/10.

7. Rastogi N, Mehra R (2013) Analysis of savitzky-golay filter for baseline wander cancellation in ecg using wavelets. International Journal of Engineering Sciences \& Emerging Technologies 6(1): 15-23.

\section{Your next submission with Juniper Publishers will reach you the below assets}

- Quality Editorial service

- Swift Peer Review

- Reprints availability

- E-prints Service

- Manuscript Podcast for convenient understanding

- Global attainment for your research

- Manuscript accessibility in different formats

( Pdf, E-pub, Full Text, Audio)

- Unceasing customer service

Track the below URL for one-step submission https://juniperpublishers.com/online-submission.php 